consumables (57.41\%) are ways to curb overcrowding. 98\% agreed that six hourly bed occupancy data from the admitting wards was important to reduce $\mathrm{ED}$ overcrowding.

Conclusion: Overcrowding in the ED is perceived to cause staff burn-out and result in poor patient outcomes. Evidencebased interventions may improve overcrowding in EDs.

Prehosp Disaster Med 2017;32(Suppl. 1):s33-s34

doi:10.1017/S1049023X17001054

Waiting Times in a Tertiary Academic Hospital Emergency Department, Iran

Elham Pishbin ${ }^{1}$, Shaghayegh Rahmani ${ }^{2}$

1. Emergency Medicine Department, Mashhad University of Medical Sciences, Mashhad/Iran

2. Imam Reza Hospital, Mashhad/Iran

Study/Objective: This project was aimed to evaluate waiting times in various processes in the $\mathrm{ED}$ of an academic hospital in Mashhad, Iran.

Background: Waiting for a physician visit in hospitals is one of the main factors associated with health care quality. Overcrowding is a significant problem in the Emergency Department (ED).

Methods: This cross sectional study was conducted in ED of our academic hospital in Mashhad, Iran. Data was gathered as a checklist. Intervals between triage and first visit, getting nursing care and discharge from ED were the main variables in our study. Data was entered using the SPSS version 16; P-value less than 0.05 was considered as significance level.

Results: In the first 25 days of the study, 1,250 patients were enrolled the study. Of those, 466 patients (37.2\%) were triaged in first and second level and 784 (62.8\%) were in third or fourth level. Mean duration between triage and first visit was $13.5 \pm 7.6$ minutes (first and second level of triage) and $16.4 \pm 10.1$ minutes (third and fourth level). Mean duration for receiving nursing care was $12.1 \pm 7.8$ minutes (first and second level of triage) and $15.6 \pm 9.5$ minutes (third and fourth level). There was a negative correlation between number of patients and waiting time for the first visit, in patients were in third and fourth level of triage $(\mathrm{p}<0.001, \mathrm{r}=0.654)$.

Conclusion: Our finding revealed that the number of ED physicians and nurses were correlated with waiting time for patients. So it seems, the use of strategies to reduce ED waiting time could be satisfying for both patients and staffs.

Prehosp Disaster Med 2017;32(Suppl. 1):s34

doi:10.1017/S1049023X17001066

Increased Burdens of Emergency Departments -

Organizational Challenges

Przemyslaw W. Gula ${ }^{1}$, Krzysztof Karwan ${ }^{2}$

1. Polish Armed Forces, Krakow/Poland

2. Polish Armed Forces, Warszawa/Poland

Study/Objective: The paper aims to assess Emergency Department (ED) organizational changes that are necessary due to constant increases of patient volume. We reviewed the ways of facilitating ED organization, and specifically the options of applying Lean analysis to optimize ED operations.
Background: The Military Institute of Medicine has one of the largest EDs in Poland, being a part of a multi-profile specialist hospital and regional trauma center for the Warsaw Agglomeration. The increase of the number of patients from 31,554 to 83,530, from 2009 to 2015 (a nationwide trend) was not matched by growth of the number of personnel or ED infrastructure, forcing change in work organization. The annual average of patients referred to the Trauma Center was 691 .

Methods: Solutions to optimize ED operations were assessed in terms of the need to adapt to the growing ED burdens, specifically based on the results of application of the Lean analysis and the International Emergency Department Leadership Institute (IEDLI) standards.

Results: During the six years under review, ED's burdens increased by $264.7 \%$, but neither the infrastructure nor the number of personnel grew accordingly, necessitating a search for organizational solutions to keep up with patient flow and ensure patient safety. The number of patients not in life-threatening condition grew from $37 \%$ to $48 \%$ of $\mathrm{ED}$ patients, proving a major challenge. Continuity of operations requires patient flow modelling, triage system modification and efforts to decrease access block. Elements of Lean analysis and IEDLI recommendations were applied to keep safety standards for patients in life-threatening conditions, and Trauma Center patients (only $0.8 \%$ of ED patients but creating long-term burden).

Conclusion: The increase of the number of ED patients who have higher expectations and create greater burdens and duties for personnel and ED infrastructure, was not matched by an increase of the number of personnel and ED infrastructure development. Therefore, it is necessary to accept solutions to reduce risk and undesirable effects.

Prehosp Disaster Med 2017;32(Suppl. 1):s34

doi:10.1017/S1049023X17001078

Risk Recognition: Rationing Emergency Department Care as a Response to Overcrowding Sandra Richardson

Christchurch Hospital Emergency Department, Canterbury District Health Board, Christchurch/New Zealand

Study/Objective: The central objective of this research is to examine the relative risks associated with rationing Emergency Department (ED) care. A number of sub-objectives are considered, including: definition and measurement of overcrowding; rationale for managing so-called 'inappropriate' attendees; definitions of primary care patients; range of risks associated with rationing of care; acknowledgment and discussion of ethical issues resulting from these.

Background: Overcrowding and the associated impact on ED flow, patient outcomes and staff recruitment, retention and morale are increasingly recognized and reported. However, a core assumption remains that much of this is due to the presence of inappropriate attendees, or those who could be treated in primary care facilities. Efforts to address this include redirection of patients away from EDs, which effectively introduces rationing of care. This has not been made explicit, nor clearly discussed in the public arena, and the allocation of risk remains unequally distributed. 
Methods: This presentation is derived from research focussing on $\mathrm{ED}$ overcrowding, including the author's $\mathrm{PhD}$ on defining ED attendance appropriateness, which utilized a Mixed Method research approach incorporating Delphi survey methodology and analysis of qualitative free text responses, as well as subsequent systematic literature reviews and ethical analysis of identified core issues. An international perspective is presented, with the use of a New Zealand health system exemplar.

Results: There are specific risks associated with current responses to patient redirection - these include clinical, professional, legal and ethical risks. These risks are disproportionately spread across the key stakeholders in this process (patients, medical staff, managers), with limited recognition of these risks.

Conclusion: There is insufficient research and audit follow-up associated with the introduction of many overcrowding mitigation processes. Tendencies exist to focus on a 'quick fix', a highly visible responses to manage primary care patients, which may not be the best use of resources.

Prehosp Disaster Med 2017;32(Suppl. 1):s34-s35

doi:10.1017/S1049023X1700108X

The Lack of Supra-Specialty/Specialty of Emergency Medicine in Greece: The Necessity, The Steps, The Problems, and the Delays Helen Pavlidou ${ }^{1}$, Leonidas Liakopoulos ${ }^{2}$, Georgios Soufras ${ }^{1}$, Agis Terzidis ${ }^{3}$, Emmanouil Pikoulis ${ }^{3}$, Theofilos Rosenberg ${ }^{3}$

1. Emergency Dpt., General Hospital of Patras, Patras/Greece

2. Health Center K. Achaia, Patras/Greece

3. Surgery Dpt., National and Kapodistrian University of Athens, Patras/Greece

Study/Objective: The reasons of necessity and the lack of Emergency Medicine Specialty in Greece.

Background: Emergency Medicine is a relatively new specialty that constantly develops all around the world. The World Health Organization (WHO) encourages governments to support the development of health services related to Emergency Care, and acknowledging the continuously increasing burden of trauma and other emergency cases. Patient visits to the Emergency Care Units are rising, mostly due to the complications of chronic diseases presented by the growing geriatric population.

Methods: We extensively reviewed the Medline-Pubmed electronic databases from 2005-2015, as well as published data in government Greek and international websites related to Emergency Medicine. In addition, we conducted online research using a small questionnaire addressed to the Greek doctors regarding their opinion about Emergency Medicine and the emergency departments. Data are included. Keywords: "Emergency Medicine Specialty;" "Emergency Departments;" "development;" "prehospital emergency medicine;" "emergency health care;" "Greece."

Results: The European Society of Emergency Medicine (EUSEM) has made special efforts to establish the specialty of Emergency Medicine (EM) in Europe, and a joint training program. Specialty or subspecialty of EM have not officially been established, although Greece is officially represented in EUSEM since 2007 by the Hellenic Society of Emergency Medicine. Training in EM is inadequate and not well organized. Recent economic crisis with subsequent frequent government changes, the lack of support from other specialties, and the cutbacks concerning health expenses have hindered the continuation of the efforts towards the recognition of the specialty.

Conclusion: Our data indicates that Greek doctors strongly support the establishment of the specialty in EM. Under these unfavorable conditions, we should continue the efforts of establishing the specialty, through integrated and documented suggestions, aiming to achieve the provision of high-quality and efficient emergency care to the patients. Improving public health is a priority of any organized society.

Prehosp Disaster Med 2017;32(Suppl. 1):s35

doi:10.1017/S1049023X17001091

\section{Turning the Heat Up on Admissions: The Impact of Extreme Heat Events on Hospital Admissions \\ Kaitlyn Porter, Judith Singleton}

School Of Clinical Sciences, Queensland University of Technology, Brisbane/QLD/Australia

Study/Objective: This study aimed to investigate the impact of extreme heat events on the admissions to the Royal Hobart Hospital (RHH), Tasmania for the period January 2003 to December 2010. The objective of this study was to determine if extreme heat events lead to an increase in hospital admissions. Background: Extreme heat events are increasing in frequency and duration and cause more deaths in Australia than any other extreme weather event. The total economic cost of extreme weather events in Australia each year is estimated at $\$ 6.3$ billion with this figure expected to double by 2030 . Extreme heat increases the number of presentations to emergency departments and the mortality and morbidity rates. Emergency departments across Australia have experienced a steady increase in presentations over the years with spikes occurring during disaster events. In 2012-2013, Tasmania had the largest percentage increase in emergency department presentations of all the Australian states; there were 147,064 presentations equating to a $3.8 \%$ increase on the previous year. This increase in public hospital emergency department presentations across Australia has led to overcrowding of emergency departments.

Methods: Non-identifiable RHH emergency department data and climate data from the Australian Bureau of Meteorology were obtained for the period 2003-2010. Statistical analysis was conducted using the computer statistical software ' $R$ ' with a Distributed Lag Nonlinear Model (DLNM) package used to fit a quasi-Poisson generalized linear regression model.

Results: The Relative Risk (RR) of admission to RHH during 2003-2010 was significant when temperatures exceeded $24^{\circ} \mathrm{C}$ $(75.2 \mathrm{~F})$. The peak effect was noted one day after an extreme heat event $(\mathrm{P}<.05)$ with a lag effect lasting 12 days. These results highlight the significant impact extreme heat events have on hospital admissions. 